\title{
Mechanisms and pharmacokinetic/pharmacodynamic profiles underlying the low nephrotoxicity and ototoxicity of etimicin
}

\author{
Lan Yao ${ }^{1}$, Jing-wei Zhang ${ }^{1}$, Bin Chen ${ }^{1}$, Ming-min Cai ${ }^{1}$, Dong Feng ${ }^{1}$, Qi-zhi Wang ${ }^{1}$, Xin-yu Wang ${ }^{1}$, Jian-guo Sun ${ }^{1}$, Yi-wen Zheng ${ }^{2,3,4}$, \\ Guang-ji Wang ${ }^{1}$ and Fang Zhou' ${ }^{1}$
}

\begin{abstract}
Etimicin (ETM), a fourth-generation aminoglycosides (AGs), is now widely clinically used in China due to its high efficacy and low toxicity. However, the mechanisms underlying its low nephrotoxicity and ototoxicity remain unclear. In the present study we compared the antibacterial and toxicity profiles of etimicin, gentamicin (GM, a second-generation AG), and amikacin (AMK, a third-generation AG), and investigated their pharmacokinetic properties in the toxicity target organs (kidney and inner ear) and subcellular compartments. We first demonstrated that ETM exhibited superior antibacterial activities against clinical isolates to GM and AMK, and it exerted minimal nephrotoxicity and ototoxicity in rats following multi-dose administration. Then, we conducted pharmacokinetic studies in rats, showed that the three AGs accumulated in the kidney and inner ear with ETM being distributed to a lesser degree in the two toxicity target organs as compared with GM and AMK high-dose groups. Furthermore, we conducted in vitro experiments in NRK-52E rat renal tubular epithelial cells and HEI-OC1 cochlear hair cells, and revealed that all the three AGs were distributed predominantly in the mitochondria with ETM showing minimal accumulation; they not only directly inhibited the activity of mitochondrial complexes IV and $\mathrm{V}$ but also inhibited mitochondrial function and its related PGC-1a-NRF1-TFAM pathway; ETM caused minimal damage to the mitochondrial complex and mitochondrial biogenesis. Our results demonstrate that the minimal otonephrotoxicity of ETM results from its lesser accumulation in mitochondria of target cells and subsequently lesser inhibition of mitochondrial function. These results provide a new strategy for discovering novel AGs with high efficacy and low toxicity.
\end{abstract}

Keywords: Aminoglycoside; etimicin; gentamicin; amikacin; nephrotoxicity; ototoxicity; pharmacokinetics; mitochondria

Acta Pharmacologica Sinica (2020) 41:866-878; https://doi.org/10.1038/s41401-019-0342-5

\section{INTRODUCTION}

Aminoglycosides (AGs) are a class of antibiotics with robust bactericidal effects. Despite the introduction of highly potent broad-spectrum antibiotics, AGs are still commonly used worldwide due to their clinical efficacy, low rate of true resistance, and low cost of therapy, especially against gram-negative bacterial infections $[1,2]$. AGs have strong polarity due to the amino and hydroxyl groups in their structures, and they are poorly absorbed via the gastrointestinal $(\mathrm{Gl})$ tract $[3,4]$. A pharmacokinetic study of AGs showed that the volume of distribution approaches the total body volume, indicating a broad distribution into tissues [5], and the binding rate of AGs to serum proteins is very low, usually less than $10 \%$ [6]. Moreover, the drugs are excreted through the kidney without metabolism $[3,4,7]$. However, severe nephrotoxicity [8] and irreversible ototoxicity [9] have seriously limited their broad usage in clinical medicine. It has been reported that AGs induce a dose-dependent nephrotoxicity in $10 \%-25 \%$ of therapeutic courses despite rigorous monitoring of serum drug concentrations and adequate fluid volume control [10]. AGinduced ototoxicity, which can result in tinnitus, vestibular disorders, and hearing loss, can occur in as many as $20 \%$ of patients who received these drugs for multiple days [9]. Although much effort has been made to understand the mechanisms of AGinduced nephrotoxicity and ototoxicity, it is still not well understood. Elucidating the mechanisms of toxicity at the cellular or subcellular level may aid in the discovery of high-efficacy and lowtoxicity AGs.

Etimicin (ETM), which belongs to the fourth generation of AGs, is a semisynthetic drug made from gentamicin C1a by introducing an ethyl group to the $1-N$ position (Supplementary Fig. S1). It is a state first-class new medicine, and there are independent intellectual property rights in China. ETM is now widely used clinically in China due to its high efficacy and low toxicity [11-13]. Currently, there is only one report showing that ETM has a better antibacterial profile than other AGs such as gentamicin, tobramycin, amikacin, and netilmicin in clinical isolates from hospitals in India [12]. One animal study also reported no significant toxicity observed in mice after ETM treatment [14]. However, it is not clear whether ETM is more effective against the clinical isolates from Chinese hospitals, and the mechanisms by which it shows lower nephrotoxicity and ototoxicity compared to those of other AGs need further clarification.

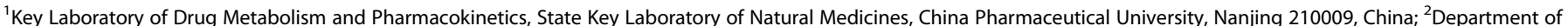

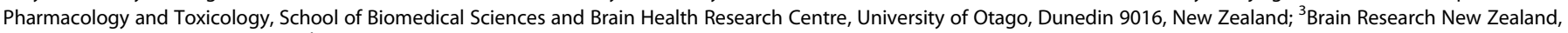
Dunedin 9016, New Zealand and ${ }^{4}$ Eisdell Moore Centre for Hearing and Balance Research, University of Auckland, Auckland 1142, New Zealand

Correspondence: Yi-wen Zheng (yiwen.zheng@otago.ac.nz) or Guang-ji Wang (guangjiwang@hotmail.com) or Fang Zhou (zf1113@163.com)

These authors contributed equally: Lan Yao, Jing-wei Zhang

Received: 17 June 2019 Accepted: 2 December 2019

Published online: 14 January 2020 
In this paper, we confirmed the superiority of the antibacterial and toxicity profiles of ETM compared to those of gentamicin (GM), a second-generation AG, and amikacin (AMK), a third-generation AG (Supplementary Fig. S1). We also studied the pharmacokinetic behaviors of these three AGs in the kidney and inner ear, which are the target organs, and subcellular compartments to elucidate the mechanisms of the low nephrotoxicity and ototoxicity of ETM.

\section{MATERIALS AND METHODS}

Chemicals and materials

Gentamicin sulfate was obtained from Jinling Pharmaceutical Co. Ltd. (Nanjing, Jiangsu, China), amikacin sulfate was obtained from Huazhong Pharmaceutical Co. Ltd. (Xiangyang, Hubei, China), and etimicin sulfate was obtained from Changzhou Fangyuan Pharmaceutical Co. Ltd. (Changzhou, Jiangsu, China). Fetal bovine serum (FBS) and Dulbecco's modified Eagle's medium (DMEM) were purchased from Gibco (Grand Island, NE, USA). Deionized water was prepared by a Milli-Q Ultrapure water purification system (Billerica, MA, USA) and was used throughout the study. The primers for $\mathrm{qPCR}$ analysis were synthesized by Invitrogen (Carlsbad, CA, USA). All other reagents were purchased from Sigma-Aldrich (St. Louis, MO, USA).

\section{Animals and treatments}

Healthy male adult Sprague-Dawley rats $(200 \pm 20 \mathrm{~g})$ were purchased from Sino-British Sippr/BK Lab Animal Ltd. (Shanghai, China) and housed in an environmentally controlled room with constant temperature $\left(22 \pm 2{ }^{\circ} \mathrm{C}\right)$ and humidity $(55 \% \pm 5 \%)$ and a $12 / 12 \mathrm{~h}$ light/dark cycle. The rats all had free access to standard laboratory rodent food and water and were kept for one week prior to the experiments in order for them to adapt to the laboratory conditions. All the animal methods were performed in accordance with the National Institutes of Health standards established in the "Guidelines for the Care and Use of Experimental Animals". All experimental protocols in animals were approved by the Ethics Committee on Animal Experimentation of China Pharmaceutical University (Nanjing, China) (2017-PKPD-09-02).

After the acclimatization period, the rats were randomly divided into five groups ( $n=6$ per group): Group 1 (Control group, vehicle aqueous saline solution i.p. administration once a day), Group 2 (GM group, $70 \mathrm{mg} / \mathrm{kg}$ GM i.p. administration once a day), Group 3 (ETM group, $70 \mathrm{mg} / \mathrm{kg}$ ETM i.p. administration once a day), Group 4 (AMK low-dose group, $70 \mathrm{mg} / \mathrm{kg}$ AMK i.p. administration once a day), and Group 5 (AMK high-dose group, $210 \mathrm{mg} / \mathrm{kg}$ AMK i.p. administration once a day). The reason for having two AMK groups is that the clinically recommended dose of AMK is approximately three times the dose of GM and ETM. The dosing solution used for intraperitoneal administration was prepared by dissolving the drug in aqueous saline solution.

For nephrotoxicity and pharmacokinetics studies, every group received a drug injection once a day for one day or seven consecutive days, and the drug was withdrawn for another seven days (Fig. 1a). The rats were sacrificed on day 2,8 , and 15 . Six animals were used for every group at every sampling time point.

For the ototoxicity study, every group received a drug injection once a day for three weeks (Fig. 1b). Auditory-evoked brainstem response (ABR) testing was performed on day 7,14 , and 21 .

\section{Tissue preparation}

For nephrotoxicity and pharmacokinetics studies, the animals were divided into three batches, and drugs were administered daily for 1 day (one batch) or 7 consecutive days (two batches). Samples were collected $24 \mathrm{~h}$ following drug administration for 1 day (day 2) and 7 consecutive days (day 8) and drug administration for 7 days with withdrawal for 7 days (day 15). The rats were sacrificed, and serum was separated from the whole blood and stored at $-80^{\circ} \mathrm{C}$ until analysis. Kidneys were immediately removed, and small portions of the upper part of the right kidney were excised and placed in 4\% paraformaldehyde and $3 \%$ glutaraldehyde for subsequent histopathology. The remainder of the right kidney and the left kidney were individually wrapped in aluminum foil, dipped in liquid nitrogen and stored at $-80^{\circ} \mathrm{C}$, pending analysis within 10 days (Fig. $1 \mathrm{a}$ ).

For the ototoxicity study, drugs were administered daily for 21 consecutive days. After sacrificing the rats, the otic capsules were immediately isolated, rapidly frozen in liquid nitrogen, and stored at $-80^{\circ} \mathrm{C}$ until further processing (Fig. 1b).

Determination of the antibacterial activities of the three AGs Thirty-six clinical strains of bacteria were obtained from the archived collection of clinical microbials of The First Affiliated Hospital of Nanjing Medical University: 6 strains of Escherichia coli (E. coli), 6 strains of Klebsiella pneumoniae (K. pneumoniae), 6 strains of Enterobacter cloacae (E. cloacae), 6 strains of Proteus mirabilis (P. mirabilis), 6 strains of Pseudomonas aeruginosa (P. aeruginosa), and 6 strains of Staphylococcus aureus (S. aureus). Minimum inhibitory concentrations (MICs) were determined by the broth microdilution method according to the Clinical and Laboratory Standards Institute (CLSI) guidelines (Clinical Laboratory Standards Institute 2009), using double strength Mueller-Hinton broth (MHB), 4x strength antibiotic solutions prepared as serial two-fold dilutions and the test organism at a concentration of $2 \times 10^{6} \mathrm{CFU} / \mathrm{mL}$. In a sterile $96-$ well plate, $100 \mu \mathrm{L}$ of double strength MHB, $50 \mu \mathrm{L}$ of each of the antibiotic dilutions and the organism suspension were mixed and incubated at $37^{\circ} \mathrm{C}$ for 18-24 h. The lowest concentration at which there was no visible growth of the organism was considered MIC. The $\mathrm{MIC}_{50}$ and $\mathrm{MIC}_{90}$ were determined as the concentrations at which $50 \%$ and $90 \%$ of the isolates were inhibited, respectively. The quality control strains recommended by the CLSI were included as internal controls throughout the study.

Nephrotoxicity evaluation following treatment with AGs Levels of blood urea nitrogen (BUN) and serum creatinine ( $\mathrm{SCr}$ ) were measured by an automatic biochemical analyzer (AU5400; Olympus Corporation, Tokyo, Japan). Paraformaldehyde-fixed renal tissue was used to examine histological changes. A portion of the renal cortex was embedded in paraffin wax blocks, sectioned into $5 \mu \mathrm{m}$ thick slices and stained with hematoxylin and eosin (H\&E) for histopathological assessment of changes due to AG-induced nephrotoxicity.

Ototoxicity evaluation following treatment with AGs

The hearing thresholds of the rats were examined with the auditoryevoked brainstem response (ABR) test once a week. The rats were anesthetized with $10 \%$ chloral hydrate $(0.3 \mathrm{~mL} / \mathrm{kg})$ and placed on a thermostatic heating pad in a sound-attenuating chamber to maintain their body temperatures at $38.5^{\circ} \mathrm{C}$. Signal generation and ABR acquisition were conducted using Tucker-Davis hardware and BioSig software (Tucker-Davis Technology system III). The stimuli consisted of tone bursts of $4,8,12,16,24$, and $36 \mathrm{kHz}$, with a duration of $10 \mathrm{~ms}$ and rise/fall of $1 \mathrm{~ms}$ (Blackman window). The signals were generated digitally using the TDT system III and delivered to the subjects through an electrostatic speaker (ES1, TDT), which presented a flat frequency response from 2 to $100 \mathrm{kHz}$. The speaker was placed $10 \mathrm{~cm}$ above the head of the subject. The sound level was calibrated using a 1/4-in. B\&K condense microphone (Mode 4349), which was placed at the position that would be occupied by the head of the animal. The output of the microphone was examined using SigCal software from TDT. The stimulation rate was $21.1 / \mathrm{s}$, and 1000 evoked responses were averaged for each trial. At each frequency, the ABR was tested by starting with a $90 \mathrm{~dB}$ sound pressure level (SPL) and then decreasing the sound amplitude in 5-10 dB steps until the threshold for detecting a repeatable response was reached. The evoked responses were recorded by subdermal electrodes, bandpass filtered between 100 and $3000 \mathrm{~Hz}$, before amplification. 
a

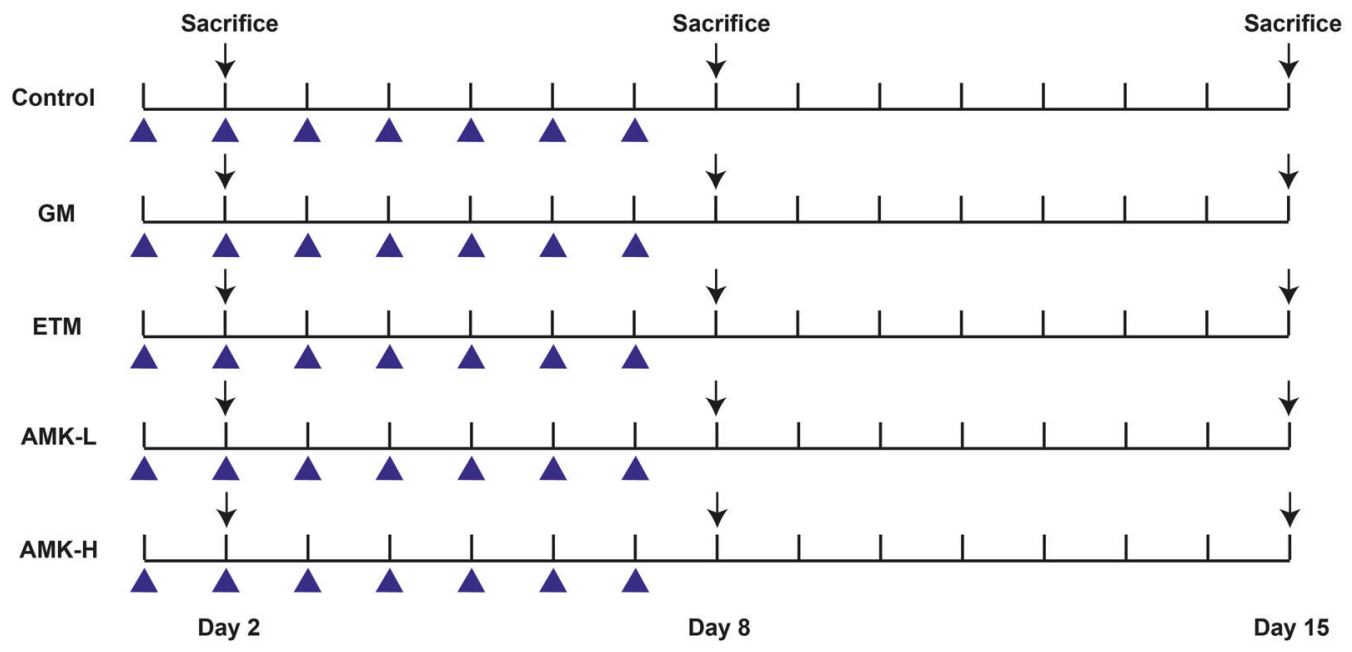

$\Delta$ : Start AGs dosing; 6 rats for every group and every time point.

b

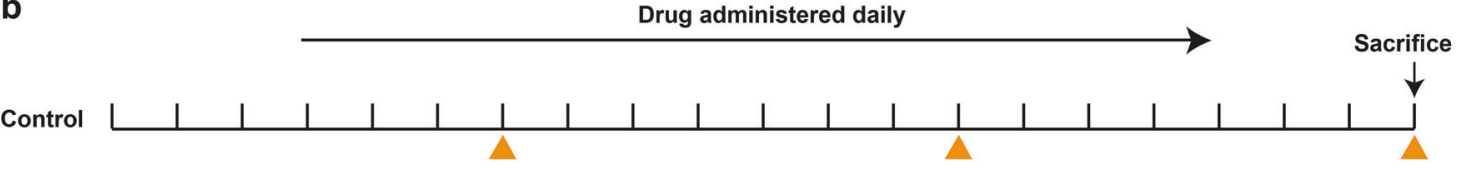

GM $L$

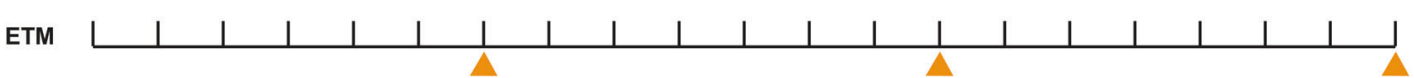
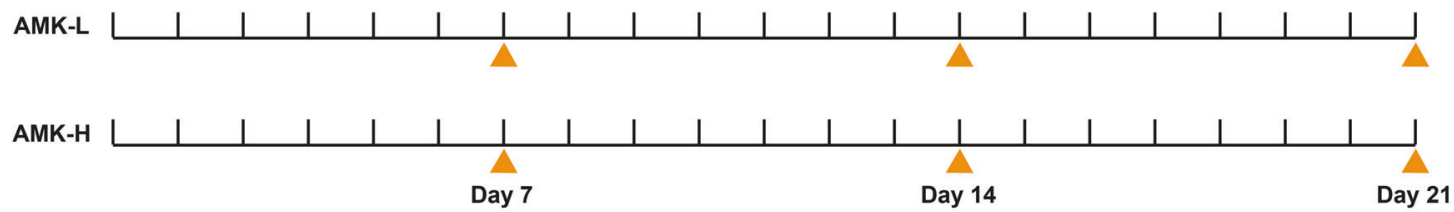

: ABR test; $n=6$.

Fig. 1 Schematic plans for the administration of aminoglycosides (AGs) and animal experiments. AG treatments and sampling for the nephrotoxicity study (a) and ototoxicity study (b). Each group at every sampling time point, $n=6$.

Pharmacokinetic studies of AGs in different rat organs

The concentrations of GM, ETM, and AMK in samples of plasma, kidney, inner ear and other organs were quantified using an LCMS/MS method as previously described [15]. Briefly, plasma and tissue homogenate with ribostamycin as an internal standard (IS) were treated with extraction solution $(10 \mathrm{mM}$ ammonium acetate, $0.4 \mathrm{mM}$ EDTA, $0.5 \% \mathrm{NaCl}$, and $2 \% \mathrm{TCA}$ ) to precipitate proteins and extract the drugs. After vortexing for $10 \mathrm{~min}$ and centrifuging at $3000 \times g$ and $4{ }^{\circ} \mathrm{C}$ for $10 \mathrm{~min}$, the supernatant was transferred to new tubes, adjusted to $\mathrm{pH} 6.5$ with $\mathrm{NaOH}(20 \%)$ or $\mathrm{HCl}(1 \mathrm{M})$, and then loaded onto an Accell Plus CM SPE cartridge. When the analytes were eluted with $2 \%$ ammonia in water, and the eluate was centrifuged at $18,000 \times g$ for $5 \mathrm{~min}$ before $10 \mu \mathrm{L}$ of the supernatant was injected into the LC-MS/MS system. The LC-MS/ MS system consisted of a Shimadzu HPLC system 20A and an AB API4000 quadrupole mass spectrometer with an electrospray ionization (ESI) interface.

Separation of AGs was carried out using a SIELC Obelisc R column $(2.1 \mathrm{~mm} \times 150 \mathrm{~mm}, 5 \mu \mathrm{m})$. The column temperature was set at $40^{\circ} \mathrm{C}$. The mobile phase consisted of solvent A ( $1 \%$ formic acid in deionized water) and solvent $B$ (acetonitrile) with the following gradient conditions: the initial proportion was $85 \%$ solvent $\mathrm{B}$, which then decreased to $2 \% \mathrm{~B}$ from 0.2 to $1 \mathrm{~min}$, holding the composition for the next $5 \mathrm{~min}$, followed by column equilibration to the initial conditions over $3 \mathrm{~min}$. The MS parameters were as follows: multiple-reaction monitoring (MRM) was used to monitor the analyte and internal standard. The selected mass transitions were $\mathrm{m} / \mathrm{z} \quad 478.4 \rightarrow 322.1$ for GM C1, $\mathrm{m} / \mathrm{z} 450.5 \rightarrow 322.1$ for GM C1a, $\mathrm{m} / \mathrm{z} 464.4 \rightarrow 160.1$ for GM C2, $\mathrm{m} / \mathrm{z} 478.3 \rightarrow 191.3$ for ETM, $\mathrm{m} / \mathrm{z} 586.6 \rightarrow 425.5$ for AMK and $\mathrm{m} / \mathrm{z}$ $455.5 \rightarrow 163.0$ for IS. The optimized collision gas, curtain gas, ion source gas 1 , and ion source gas 2 (all nitrogen) were set at 12, 30, 45, and 65 Arb, respectively. The ion spray voltage was $4500 \mathrm{~V}$, and the source temperature was maintained at $450^{\circ} \mathrm{C}$. Data acquisition and analysis were performed using Analyst TF 1.5.1 software (SCIEX, Framingham, MA, USA).

Cell culture and treatments

The rat proximal renal tubular cell line (NRK-52E) was purchased from American Type Culture Collection (Rockville, MD, USA). The House Ear Institute-Organ of Corti 1 cell line (HEI-OC1), which is derived from the cochlea of the Immortomouse and was characterized by Kalinec et al. [16], was kindly gifted by 
Dr. Ren-jie Chai from the MOE Key Laboratory of Developmental Genes and Human Disease of the Institute of Life Sciences at Southeast University, China. Cells were cultured in DMEM supplemented with $10 \%$ FBS and $100 \mathrm{IU} / \mathrm{mL}$ penicillin (Life Technologies, Carlsbad, CA, USA) and were grown in an atmosphere of $5 \% \mathrm{CO}_{2}$ at $37^{\circ} \mathrm{C}$. The cells were divided into five groups: (1) control (serum-free medium) cells; (2) GM-treated (3 mM) cells; (3) ETM-treated (3 mM) cells; (4) AMK low concentration-treated (3 $\mathrm{mM})$ cells; and (5) AMK high concentration-treated $(9 \mathrm{mM})$ cells. The cells were incubated with AGs at $37^{\circ} \mathrm{C}$ for $48 \mathrm{~h}$.

\section{Subcellular localization of AGs}

To quantitatively analyze the subcellular distribution of AGs, a cell fractionation approach [17] was used to separate nuclei, cytoplasm, and mitochondria from NRK-52E and HEI-OC1 cells. Concentrations of AGs within each subcellular compartment were determined using LC-MS/MS as described above and were normalized based on initial dosing volume and protein content. All experiments were conducted in triplicate.

\section{Examination of mitochondrial ultrastructure using transmission} electron microscopy

For electron microscopy, samples of the renal cortex were fixed in $3 \%$ glutaraldehyde in $0.1 \mathrm{M}$ cacodylate buffer solution $(\mathrm{pH} 7.4)$ and then extensively washed with PBS before post-fixation in $1 \%$ osmium tetroxide phosphate buffer solution. The samples were then dehydrated in a graded series of ethanol and embedded in epoxide resin. Ultrathin sections ( $80 \mathrm{~nm}$ thickness) were cut with a diamond knife on an LKB Ultratome IV (LKB Instruments, Bromma, Sweden), mounted on grids, stained with uranyl acetate and lead citrate, and examined under a Hitachi 7500 electron microscope (Hitachi, Japan). All histological examinations were performed by an experienced pathologist who was blinded to the experimental groups.

Intracellular reactive oxygen species (ROS) and mitochondrial superoxide measurement

NRK-52E cells and HEI-OC1 cells were cultured in 6-well plates. After treatment with AGs, the cells were incubated with $10 \mu \mathrm{M}$ $2^{\prime}, 7^{\prime}$-dichloro-fluorescein diacetate (DCFH-DA) for $30 \mathrm{~min}$, which is a cell permeable reagent that can be oxidized by ROS and become highly fluorescent. Cells were then harvested and analyzed in triplicate using a Powerwave 200 microplate reader (Bio-Tek Instruments, Winooski, VT, USA) at a wavelength of $488 / 535 \mathrm{~nm}$. For mitochondrial superoxide detection, the cells were incubated with $5 \mu \mathrm{M}$ Mito-Sox Red at $37^{\circ} \mathrm{C}$ for $10 \mathrm{~min}$ in the dark, harvested and analyzed with a microplate reader at an excitation/emission of $510 / 580 \mathrm{~nm}$. The values were normalized to the protein levels in each sample, which were determined by a BCA protein assay.

Detection of intracellular ATP levels

Intracellular ATP levels were determined using an enhanced ATP assay kit (Beyotime, China). Lysed cells were centrifuged at $12,000 \times g$ for 5 min at $4{ }^{\circ} \mathrm{C}$, and the supernatant was collected. A total of $100 \mu \mathrm{L}$ of detecting solution was added to a 96-well plate and incubated at room temperature for $5 \mathrm{~min}$ before the supernatant $(20 \mu \mathrm{L})$ was added to the plate, mixed, and read within $30 \mathrm{~min}$. Total ATP levels were calculated from the standard curve that was generated from serial concentrations of standard solution on each plate. The results were normalized to the protein concentration in each sample.

Measurement of mitochondrial electron transport chain (ETC) complex activity

Direct inhibition of the five ETC complexes by AGs was measured with the MitoTox Complete OXPHOS Activity Assay Panel (Abcam, Cambridge, MA, USA), following the manufacturer's protocol. Briefly, GM, ETM, and AMK were diluted to various concentrations in the appropriate assay solution and directly added to each of the five
OXPHOS complexes, which were captured from isolated bovine heart mitochondria in their functionally active states with highly specific monoclonal antibodies attached to 96-well microplates. A specific inhibitor of each of the five complexes was used as a positive control: rotenone (Complex I, $17.3 \mathrm{nM}$ ), thenoyltrifluoroacetone (TTFA, Complex II, $30 \mathrm{mM}$ ), antimycin A (Complex III, $22 \mathrm{nM}$ ), KCN (Complex IV, $3.2 \mathrm{mM}$ ), and oligomycin (Complex V, 8 nM). Each specific inhibitor was also tested on other complexes as negative controls for potential off-target effects (for example, rotenone was a negative control for complexes II to V). Microplate wells coated with a null capture antibody (Complexes I, II, IV, and V) or wells without mitochondria (Complex III) were used as background controls. After the addition of the assay solution-diluted AGs to the 96-well microplates, the absorbance was immediately measured in each well using a microplate reader (Bio-Tek Instruments, Winooski, VT, USA). The wavelengths were set at $340 \mathrm{~nm}$ for Complex I and V, $600 \mathrm{~nm}$ for Complex II, and $550 \mathrm{~nm}$ for Complex III and IV, and the measurements were taken every $60 \mathrm{~s}$ for $2 \mathrm{~h}$ for Complex I, every $60 \mathrm{~s}$ for $1 \mathrm{~h}$ for Complex II, IV, and V and every $20 \mathrm{~s}$ for $5 \mathrm{~min}$ for Complex III. The activity of each complex was determined by the rate of change in absorbance after background subtraction.

Real-time PCR and quantification of mitochondrial DNA (mtDNA) copy number

Total RNA was isolated using the TRlzol method in accordance with the manufacturer's instructions and quantified using ultraviolet spectrophotometry. Complementary DNA was synthesized with the PrimeScript RT Reagent Kit Perfect Real Time (Takara, Japan). The primer sequences are shown in Supplementary Table S1. PCR was performed using SYBR green PCR mastermix in a CFX96 real-time RTPCR detection system (Bio-Rad, USA). The relative expression levels of the detected genes were normalized to $\beta$-actin expression.

For the quantification of mtDNA copy number, total DNA was extracted using the MiniBEST Universal Genomic DNA Extraction Kit (TaKaRa, Japan). We compared the relative amounts of mtDNA with the nuclear DNA content. The mtDNA amplicons were generated from a Complex IV segment, and the nuclear amplicons were generated through amplification of a GAPDH segment, as previously described [18]. The threshold cycle number $(\mathrm{Ct})$ values of $\mathrm{mtDNA}$ and GAPDH were determined for each individual quantitative PCR run. The ddCt (mtDNA to GAPDH) represented the mtDNA copy number in each cell.

\section{Western blotting analysis}

Proteins $(50 \mu \mathrm{g})$ in the whole cell lysate were separated by $10 \%$ SDS-polyacrylamide gel and transferred to polyvinylidene fluoride membranes (Bio-Rad, Hercules, CA, USA). After blocking with 5\% nonfat milk, the membranes were incubated with anti-PGC-1a antibody (1:1000; Novus, Littleton, CO, USA), anti-NRF1 antibody (1:1000; Proteintech, Rosemont, IL, USA), anti-TFAM antibody (1:500; Santa Cruz, Dallas, TX, USA) or anti- $\beta$-actin antibody (1:1000; Cell Signaling Technology, Danvers, MA, USA) overnight at $4{ }^{\circ} \mathrm{C}$, followed by incubation with horseradish peroxidase (HRP)linked anti-mouse or anti-rabbit secondary antibodies (1:10,000; Cell Signaling Technology, MA, USA) for $1 \mathrm{~h}$ at $37^{\circ} \mathrm{C}$. The signals were detected with an enhanced chemiluminescence kit (Thermo Fisher Scientific, Waltham, MA, USA) and captured with a ChemiDoc $\mathrm{XRS}^{+}$System (Bio-Rad, Hercules, CA, USA).

\section{Statistical analysis}

All data are expressed as the mean \pm SEM. A two-way ANOVA followed by a Newman-Keuls post hoc test was performed on measurements of nephrotoxicity indexes, $A B R$ thresholds and PGC-1a-NRF1-TFAM mRNA and protein levels. A two-tailed, unpaired Student's $t$-test was performed when comparing two groups, and a one-way ANOVA followed by a Dunnett's multiple comparisons test was used when comparing more than two groups. A value of $P<0.05$ was considered significant. 


\section{RESULTS}

ETM had the strongest antibacterial activity against clinical isolates among the three AGs

Table 1 depicts the comparative MICs required to inhibit 50\% and $90 \%$ of the clinical isolates from Chinese hospitals that were tested in the study. The $\mathrm{MIC}_{50}$ and $\mathrm{MIC}_{90}$ of ETM against E. coli, $K$. pneumoniae, $E$. cloacae, $P$. mirabilis, and $P$. aeruginosa were significantly lower than those of GM and $A M K$, indicating that ETM was more potent. Although the $\mathrm{MIC}_{50}$ of ETM $(1.25 \mu \mathrm{g} / \mathrm{mL})$ was marginally higher than that of GM $(0.75 \mu \mathrm{g} / \mathrm{mL})$, the $\mathrm{MIC}_{90}$ of ETM was at least two times lower than those of GM and AMK for grampositive S. aureus.

ETM exhibited minimal nephrotoxicity in rats

The body weights of rats treated with saline and the three AGs were monitored daily. Compared with the body weight of the control group, the body weights were significantly lower in the GM and AMK high-dose (AMK-H) groups at day $8(P<0.001)$ and day $15(P<0.001)$, while there was no significant difference between the control and ETM groups. After drug treatment for seven days, the body weight of the control group was $234.5 \pm 2.0$ $\mathrm{g}$ at day 8 and $282.8 \pm 1.8 \mathrm{~g}$ at day 15 after drug withdrawal. The GM group showed minimal body weight at day $8(200.2 \pm 2.6 \mathrm{~g})$ and at day $15(227.4 \pm 2.7 \mathrm{~g})$. The AMK low-dose (AMK-L) group $(229.1 \pm 2.6$ and $249.4 \pm 5.0 \mathrm{~g}, P<0.01)$ and the ETM group $(237.2$ \pm 2.6 and $278.8 \pm 5.4 \mathrm{~g}, P<0.001$ ) had significantly higher body weights than those of the GM group. It is noteworthy that treatment with ETM caused no significant changes in body weight compared with that of the control group. The order of body weights from low to high was GM group $\approx A M K-H$ group $<$ AMK-L group < ETM group < control group (Fig. 2a).

GM caused a marked reduction in renal function compared to that of the control group, indicated by significant increases in BUN and $\mathrm{SCr}$ levels at day 8 after drug treatment $(P<0.001$, Fig. $2 \mathrm{~b})$. However, renal function in both the ETM and AMK-L groups was similar to that of the control group and was significantly better than that of the GM group $(P<0.001$, Fig. 2b). The gross anatomical assessment on day 8 showed that the kidneys of the GM and AMK-H groups had severe hyperemia and edema, while the heart, liver, and lung were normal (data not shown). Histological sections of kidneys from day 8 were examined under a microscope, and the degree of kidney damage was scored using the International Harmonization of Nomenclature and Diagnostic Criteria for Lesions in Rats and Mice (INHAND). As shown in Fig. 2c and Table 2, the control group, as well as the ETM and AMK-L groups, showed normal tubular structures $(-)$, whereas marked tubule injury, massive necrosis, infiltration of inflammatory cells, and intraluminal hyaline casts were observed in the kidney sections of the GM group $(+++)$. The ETM and AMK-L groups showed a mild degree of histopathological changes in kidney sections, which was similar to that of the control group, while the AMK-H group displayed moderate injury. Because kidney injury molecule-1 (KIM-1) and neutrophil gelatinase-associated lipocalin (NGAL) are regarded as early markers of renal injury [19, 20], the mRNA expression of these two markers in the kidney was measured by qPCR. The levels of these two markers in both the ETM and AMK-L groups were not significantly different from those in the control group at day 8, while marked increases were observed in the GM group (11.8-fold and 12.3-fold of the control group). KIM-1 and NGAL gene expression in the AMK-H group trended higher than in the control group but did not reach a statistically significant difference (Fig. 2d).

On day 15 (one week after drug withdrawal), the previously mentioned nephrotoxicity indexes were examined again. All nephrotoxicity indexes showed partial recovery. The BUN and $\mathrm{SCr}$ concentrations in all four treatment groups recovered to approximately the level of the control group. Only the SCr level of the GM group showed a significant difference from that of the control 

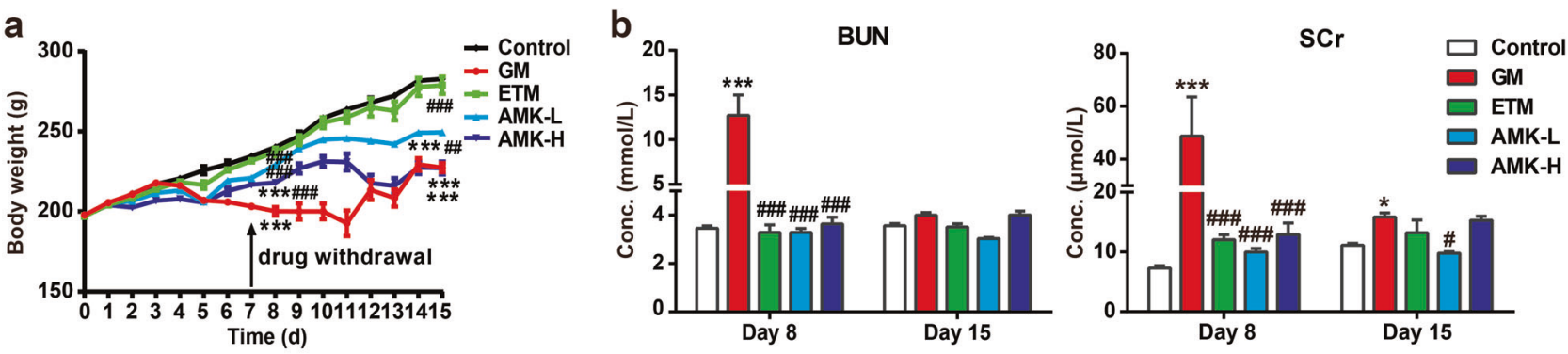

C
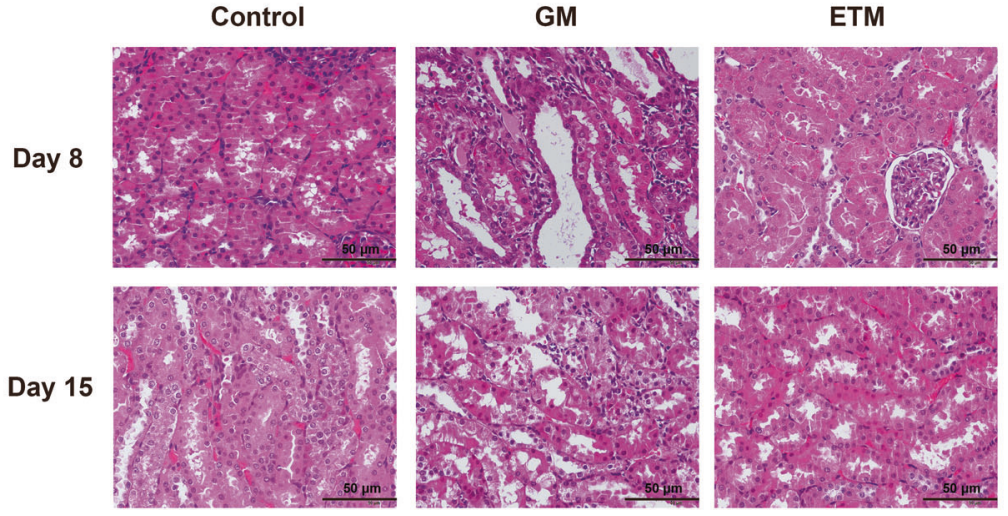

AMK-L

AMK-H

d

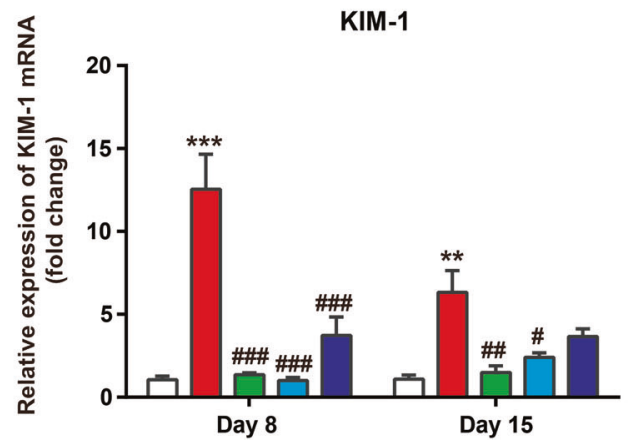

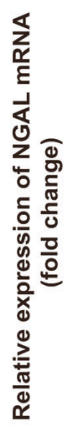

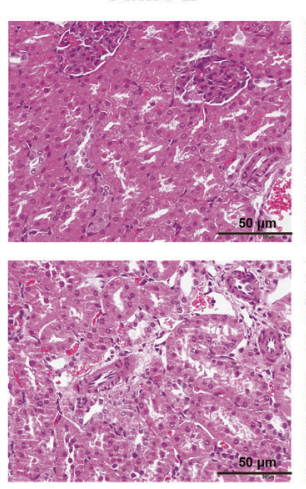

NGAL

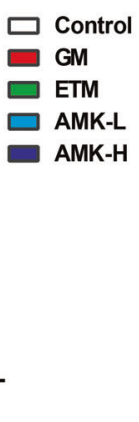

Fig. 2 Indexes of kidney dysfunction measured $24 \mathrm{~h}$ following AG administration for 7 days (day 8 ) and withdrawal for 7 days (day 15). a Body weights of rats in the studied groups; b blood urea nitrogen (BUN) and serum creatinine ( $\mathrm{SCr}$ ) in the studied rat groups; $\mathbf{c}$ photomicrographs of kidney tissue sections stained with H\&E ( $\times 200)$. Scale bar: $50 \mu \mathrm{m}$; d effect of AG treatments on kidney injury molecule-1 (KIM-1) and neutrophil gelatinase-associated lipocalin (NGAL) gene expression in the studied rat groups. Gentamicin (GM): $70 \mathrm{mg} / \mathrm{kg}$ once daily i.p. for 7 days. Etimicin (ETM): $70 \mathrm{mg} / \mathrm{kg}$ once daily i.p. for seven days. Amikacin low-dose (AMK-L): $70 \mathrm{mg} / \mathrm{kg}$ once daily i.p. for 7 days. Amikacin high-dose (AMK-H): $210 \mathrm{mg} / \mathrm{kg}$ once daily i.p. for 7 days. The results are presented as the means \pm SEM $(n=6) .{ }^{*} P<0.05,{ }^{*} P<0.01$, ${ }_{* * *} P<0.001$ vs. control group. ${ }^{\#} P<0.05,{ }^{\# \#} P<0.01$, \#\#\# $P<0.001$ vs. GM group.

Table 2. Degrees of histopathological changes in kidney sections of different experimental groups.

\begin{tabular}{|c|c|c|c|c|c|c|c|c|}
\hline \multirow[t]{2}{*}{ Groups } & \multicolumn{2}{|c|}{ Tubular necrosis } & \multicolumn{2}{|c|}{ Hyaline casts in tubular lumen } & \multicolumn{2}{|c|}{ Tubular degeneration } & \multicolumn{2}{|c|}{$\begin{array}{l}\text { Cellular inflammatory } \\
\text { infiltration }\end{array}$} \\
\hline & Day 8 & Day 15 & Day 8 & Day 15 & Day 8 & Day 15 & Day 8 & Day 15 \\
\hline Control group & - & - & - & - & - & - & - & - \\
\hline GM group & +++ & ++ & ++ & + & +++ & ++ & +++ & ++ \\
\hline ETM group & - & - & - & - & + & - & + & + \\
\hline AMK-L group & - & - & - & - & + & + & + & + \\
\hline
\end{tabular}

group on day 15. After drug withdrawal, histopathological changes in kidney sections were all improved. In the GM group, tubular injury, necrosis, cellular inflammatory infiltration, and intraluminal hyaline casts were substantially alleviated after drug withdrawal. No renal tubular injury was observed in the ETM group, but mild mononuclear cell infiltration was observed. There was no significant difference in KIM-1 and NGAL gene expression between the ETM and control groups. The KIM-1 and NGAL gene expression in the GM group decreased from 11.8-fold and 12.3-fold of the control group on day 8 to 5.8 -fold and 5.9-fold of the control group on day 15. The nephrotoxicity index of the ETM group was similar to that of the control group (Fig. 2; Table 2). 
872

ETM exhibited minimal ototoxicity in rats

Since the rate of AG-induced cochlear toxicity is slower in adult animals than in neonates [21], we monitored the auditory-evoked brainstem response (ABR) thresholds for three weeks following drug treatment. There were no significant threshold changes in the first week for all groups (Fig. 3a). By the second week (Fig. 3b), the ABR thresholds of the GM and AMK-H groups were significantly increased $(P<0.05)$ compared with those of the control group, especially when the rats were stimulated by high frequencies $(P<0.001)$. The increased threshold change in the $\mathrm{GM}$ group was higher than that of the AMK-H group. In contrast, the ABR thresholds of the ETM and AMK-L groups were similar to those of the control group, indicating no significant toxic effect on rat auditory function. After three weeks of treatment (Fig. 3c), the increased $A B R$ thresholds in the GM group became much higher than those in the other groups. The ABR thresholds of the ETM and AMK-L groups were not significantly different from the control group.
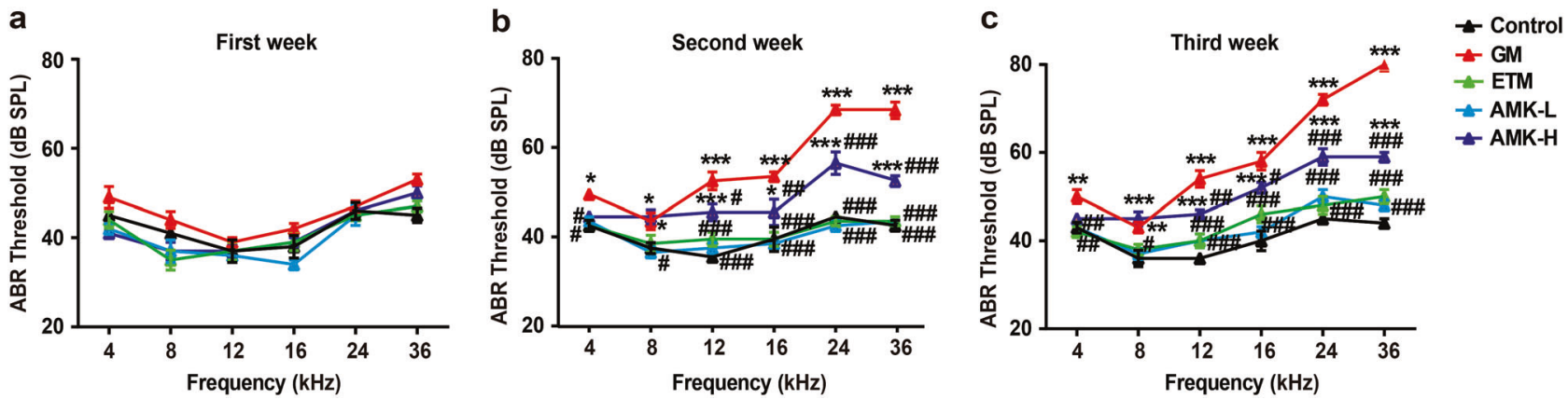

Fig. 3 Hearing loss in AG-treated rats. Auditory-evoked brainstem response (ABR) measurements were performed on rats injected with saline or AGs once daily for 1 week (a), 2 weeks (b), and 3 weeks (c). The results are presented as the means \pm SEM $(n=6)$. ${ }^{*} P<0.05, * * P<0.01$, ${ }^{* * *} P<0.001$ vs. control group. ${ }^{\#} P<0.05,{ }^{\# \#} P<0.01, " \# \# P<0.001$ vs. GM group.

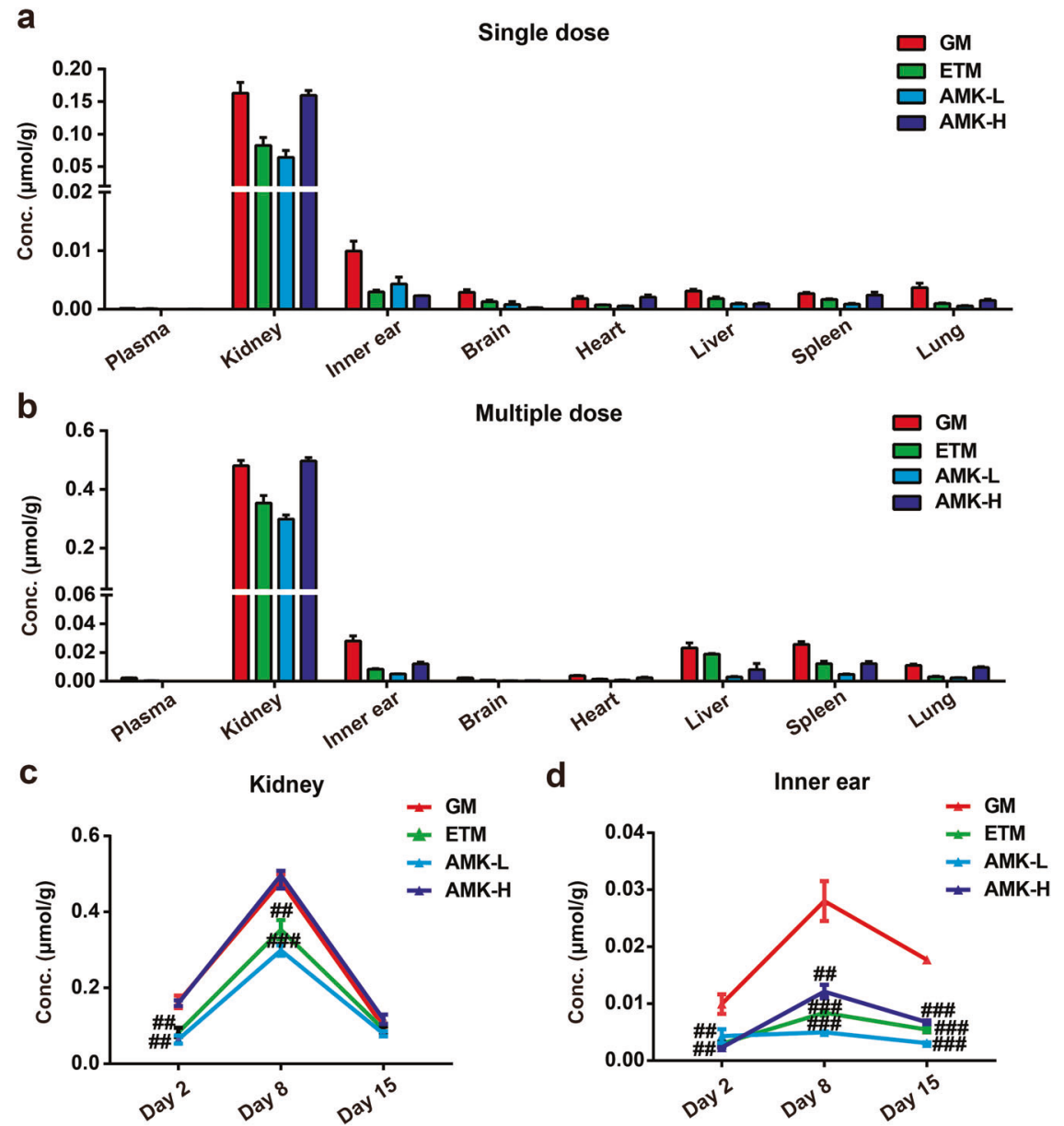

Fig. 4 Pharmacokinetic studies of AGs in vivo. AG concentrations in rat plasma and tissues after a single dose (a) and multiple doses for 7 days (b). AG concentrations measured $24 \mathrm{~h}$ following AG administration for 1 day (day 2) and 7 days (day 8 ) and withdrawal for 7 days (day 15 ) in rat kidney (c) and inner ear (d). The GM, ETM, and AMK-L groups were administered an i.p dose of $70 \mathrm{mg} / \mathrm{kg}$, and the AMK-H group was administered an i.p. dose of $210 \mathrm{mg} / \mathrm{kg}$. The results are presented as the means \pm SEM $(n=6) . ~ \# \# P<0.01,{ }^{\# \#} P<0.001$ vs. GM group. 


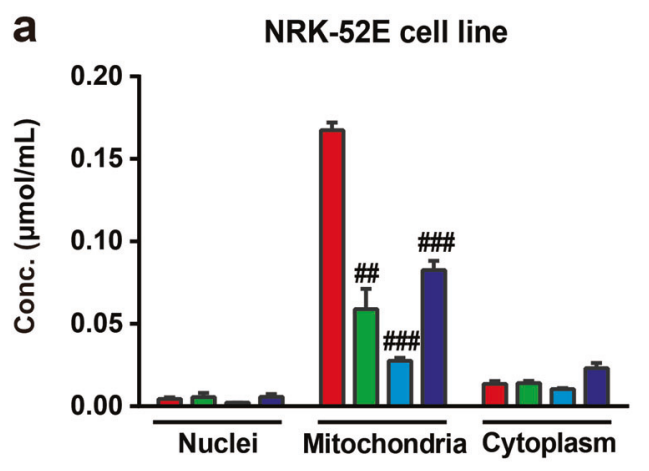

b

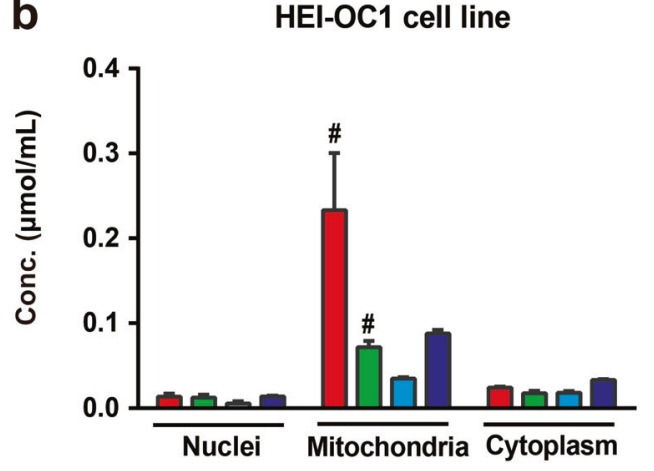

Fig. 5 Subcellular localization in vitro of AGs. AG concentrations in nuclei, mitochondria, and cytoplasm after treatment for $48 \mathrm{~h}$ in NRK52E (a) or HEI-OC1 cells (b). The GM, ETM, and AMK-L groups were treated at a concentration of $3 \mathrm{mM}$; the AMK-H group was treated at a concentration of $9 \mathrm{mM}$. The results are presented as the means \pm SEM $(n=6) .{ }^{\#} P<0.05,{ }^{\# \#} P<0.01,{ }^{\# \# \#} P<0.001$ vs. GM-treated group.

ETM showed minimal accumulation in the kidney and inner ear Twenty-four hours after a single dose administration, seven days after multidose treatment or seven days after drug withdrawal, tissue samples were collected, and drug concentrations were measured by using LC-MS/MS. The results showed that AG concentrations in the kidney were much higher than those in the plasma and other tissues and that the concentrations in the inner ear were much higher than those in the plasma (Fig. 4a, b). After repeated administration for seven consecutive days, the AGs accumulated in the kidney and inner ear. In detail, drug concentrations in the kidney increased 2.9 to $4.7-$ fold, while those in the inner ear increased 1.2 to 5.3 -fold (Fig. 4c, d). Moreover, on day 8 , the drug concentrations in the kidney and inner ear showed the same trend as the nephrotoxicity and ototoxicity results, which was supported by the observation that the GM group showed a higher level of drug accumulation $(P<0.01$, Fig. $4 c, d)$ and toxicity (described above) than those of the ETM and AMK-L groups. After AG withdrawal for seven days, the drug concentrations in the kidney decreased largely, and no significant differences were observed among the GM, ETM, and AMK groups (Fig. 4c). The amount of drug in the inner ear decreased slightly, which was almost consistent with that on day 8 (Fig. 4d).

AGs were mainly distributed in the mitochondria of renal tubular cells and inner ear hair cell-like cells, and ETM had minimal accumulation

To investigate where AGs go after they enter the target cells in the kidney and inner ear, the NRK-52E rat proximal renal tubular cell line and the HEI-OC1 organ of Corti cell line were used to quantitatively analyze the subcellular distribution of AGs. As shown in Fig. 5, GM, ETM, and AMK were all predominantly distributed in mitochondria, rather than the nuclei and cytoplasm (Fig. 5a, b), which suggested that mitochondria might be direct toxicity targets of AGs. Interestingly, ETM and AMK-L, which demonstrated lower toxicity, exhibited a lower concentration in the mitochondria compared with that of GM $(P<0.05)$.

AGs induced mitochondrial dysfunction, and ETM caused minimal mitochondrial damage

To investigate whether the accumulation of AGs in the mitochondria could lead to mitochondrial ultrastructural damage, kidney tissues from AG-treated rats were examined using electron microscopy. Typical mitochondria with normal intact cristae and dense matrices were observed in the control group. The GM and AMK-H groups displayed swollen mitochondria with disorganized and fragmented cristae. In contrast, the mitochondrial ultrastructure of the ETM group displayed no significant changes and was similar to that of the control group (Fig. 6a).

To further evaluate the effects of AGs on mitochondrial function, several independent endpoints were measured in NRK-52E and HEIOC1 cells, including reactive oxygen species (ROS) levels, mitochondrial superoxide levels, mitochondrial membrane potential (MMP), mitochondrial DNA (mtDNA) copy number, and ATP levels (Fig. 6b, c). ROS levels in the $3 \mathrm{mM}$ GM-treated group increased to almost 1.7times higher than those of the control group, while the $3 \mathrm{mM}$ ETMtreated and $3 \mathrm{mM}$ AMK-treated groups had lower ROS levels than those of the GM-treated group. The levels of ROS and mitochondrial superoxide production from low to high were control group $\approx$ or $<3 \mathrm{mM}$ ETM-treated group $<3 \mathrm{mM}$ AMK-treated group $<9 \mathrm{mM}$ AMK-treated group $\approx 3 \mathrm{mM}$ GM-treated group. MMP, mtDNA copy number, and ATP levels were significantly reduced in the $3 \mathrm{mM} \mathrm{GM}$ and $9 \mathrm{mM}$ AMK-treated groups, while the $3 \mathrm{mM}$ ETM-treated group displayed a minimal reduction. The levels of MMP, mtDNA copy number, and ATP from high to low were control group $>3 \mathrm{mM}$ ETMtreated group $\approx$ or $>3 \mathrm{mM}$ AMK-treated group $>9 \mathrm{mM}$ AMK-treated group $\approx 3 \mathrm{mM} \mathrm{GM}$-treated group.

AGs inhibited mitochondrial electron transport chain (ETC) complexes, and ETM caused minimal inhibition

It has been demonstrated that mitochondrial toxicity is closely related to the inhibition of mitochondrial ETC complex activity $[22,23]$. Our results showed that GM and AMK but not ETM inhibited complex I activity in a concentration-dependent manner, and the inhibitory effect of GM was the highest among the three AGs. At the highest test concentration of $500 \mu \mathrm{M}, \mathrm{GM}$ and AMK inhibited complex I activity up to $85 \%$ and $50 \%$, respectively, while concentrations of ETM up to $500 \mu \mathrm{M}$ caused less than $10 \%$ inhibition (Fig. 7a). As shown in Fig. 7b, c, complexes II and III were affected to a much lower extent by the three AGs, with less than $20 \%$ inhibition of complex II and less than $30 \%$ inhibition of complex III. In contrast, all three AGs induced inhibition of complexes IV and V. When the concentration of GM and AMK was higher than $20 \mu \mathrm{M}$, the inhibition of complexes IV and $\mathrm{V}$ was greater than 50\%; at the highest concentration of $500 \mu \mathrm{M}$, the inhibition of these complexes was close to $100 \%$. These inhibitory effects of GM and AMK were concentration-dependent. Within the range of concentrations tested, ETM inhibited complexes IV and V without significant concentration dependence, and the inhibition only reached $50 \%$ at the highest test concentration of $500 \mu \mathrm{M}$ (Fig. 7d, e).

ETM exerted less inhibition of the PGC-1a-NRF1-TFAM pathway than other AGs

Scarpulla et al. [24] reported that the PGC-1a-NRF1-TFAM pathway can influence mitochondrial biogenesis by regulating mtDNA content. Since we found that AGs caused a reduction in mtDNA copy number, it is conceivable that AGs may also affect the PGC-1aNRF1-TFAM pathway. Our results demonstrated that treatment of NRK-52E and HEI-OC1 cells with $3 \mathrm{mM} \mathrm{GM}$ and $9 \mathrm{mM}$ AMK for $48 \mathrm{~h}$ resulted in a significant decrease in the expression of PGC-1a, NRF1, and TFAM at both the mRNA (Fig. 8a) and protein levels (Fig. 8b), 
Control

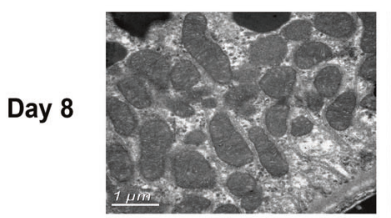

Day 15

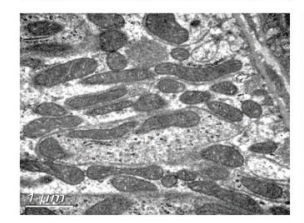

GM
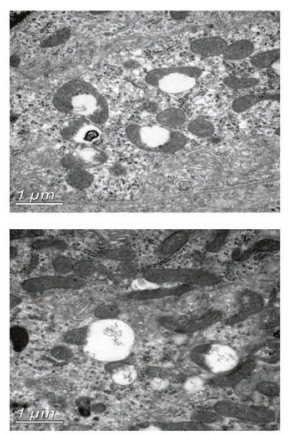

ETM
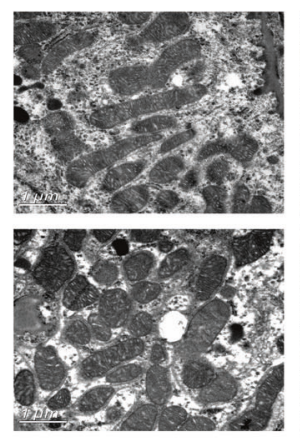

AMK-L
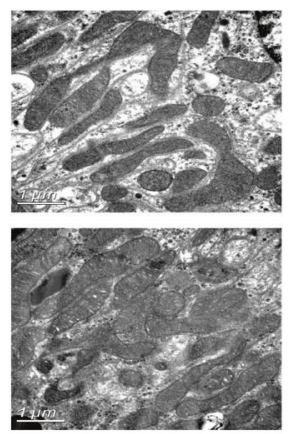

AMK-H
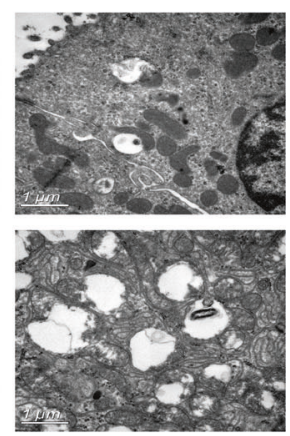

b NRK-52E:
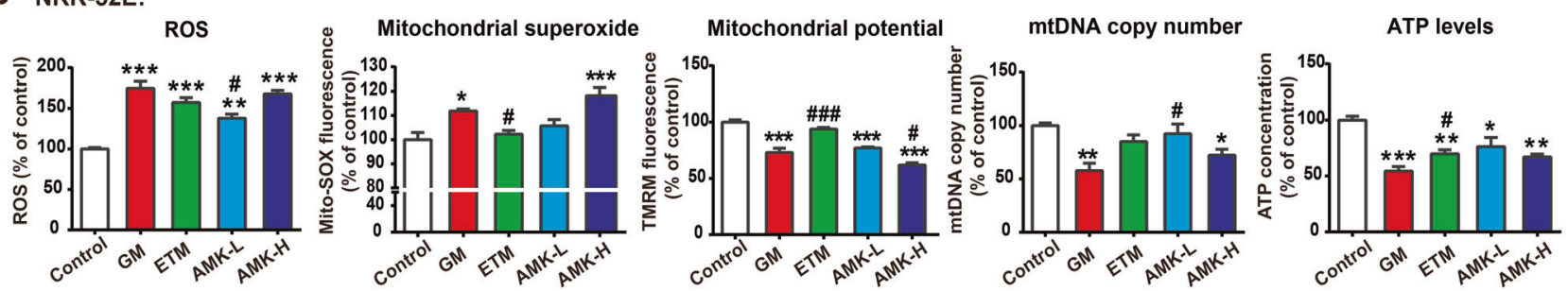

C HEI-OC1:
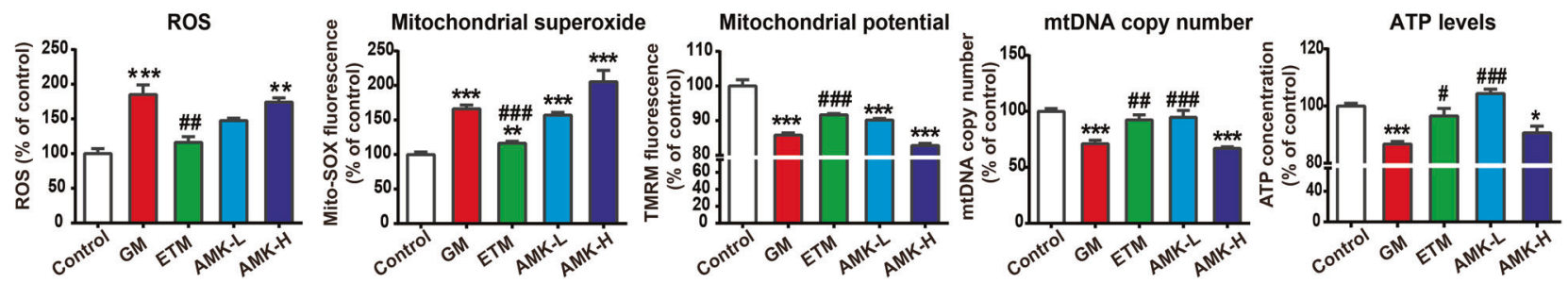

Fig. 6 AG treatment induces mitochondrial dysfunction. a Effects of AGs on the mitochondrial structure and shape of the kidney. Scale bar: $1 \mu \mathrm{m}$; b reactive oxygen species (ROS) levels, mitochondrial superoxide levels, mitochondrial membrane potential (MMP), mitochondrial DNA ( $m t D N A)$ copy number, and ATP levels were measured in NRK-52E cells; c ROS levels, mitochondrial superoxide levels, MMP, mtDNA copy number, and ATP levels were measured in HEI-OC1 cells. The GM, ETM, and AMK-L groups were treated at a concentration of 3 mM for 48 h; the AMK-H group was treated at a concentration of $9 \mathrm{mM}$ for $48 \mathrm{~h}$. The results are presented as the means \pm SEM $(n=6)$. ${ }^{*} P<0.05,{ }^{*} P<0.01$, ${ }^{* * *} P<0.001$ vs. control group. ${ }^{\#} P<0.05,{ }^{\# \#} P<0.01,{ }^{\# \# \#} P<0.001$ vs. GM-treated group.

while ETM exerted the least inhibition among the three AGs. This result was consistent with the change in mtDNA copy number.

\section{DISCUSSION}

In previous years, the use of AGs has been in decline due to the potential for serious adverse effects. However, the recent global spread of resistant pathogens has led to a resurgence of interest in AG clinical use. Therefore, it is essential to introduce new AGs with high efficacy and low toxicity. In this work, we examined the mechanisms of the low nephrotoxicity and ototoxicity of ETM, which is the newest AG (the fourth generation) invented in China. ETM exhibited very good antibacterial activity against tested clinical isolates from Chinese hospitals and was more effective than GM and AMK. The pharmacokinetic behaviors of these three AGs in the toxicity targets of the kidney and inner ear were investigated to test the contribution potential of pharmacokinetic differences to the low nephrotoxicity and ototoxicity of ETM.

Previous studies have demonstrated that AMK is less toxic than GM by using several renal and auditory functional measurements $[25,26]$. Our in vivo pharmacodynamic studies supported the former results and confirmed that ETM showed minimal nephrotoxicity and ototoxicity when compared with those of GM and AMK. It was noted that AGs, especially GM and high-dose administration of AMK daily for 7 days, caused massive injury in the kidneys that was observed biochemically and histopathologically, as well as the upregulation of KIM-1 and NGAL mRNA levels. In contrast, the damage from the ETM treatment was slight. Comparative research on toxicity should be performed among the control group, GM group, ETM group and AMK high-dose group, for they were correlated with clinical dose. On day 15 (1 week after drug withdrawal), all nephrotoxicity indexes showed partial recovery, and these indexes in the ETM group were the closest to those of the control group. For ototoxicity, AGs have been shown to significantly enhance ABR threshold shifts in animals [27]. Similarly, in this study, we found that AGs induced hearing injury in rats. The GM group had the most severe injury to rat auditory function, while no significant toxic effect was observed in the ETM group.

The present study also showed that AGs accumulated in the kidney and inner ear, which was in line with previous studies [28-30]. It is noteworthy that a higher drug accumulation in toxicity target organs correlated with a higher toxicity, which was shown by the pharmacokinetic and toxicity studies of the three AGs on day 8. This may explain, at least in part, the low nephrotoxicity and ototoxicity of ETM. Then, we explored the subcellular distribution of AGs. Although there have been studies that focused on the subcellular distribution of GM in bullfrog saccular hair cells [31] and rat renal proximal tubular cells [32], the drug concentration within each subcellular compartment was not reported, and a comparative analysis with these other drugs was lacking. Our pharmacokinetic results showed that AGs mainly 

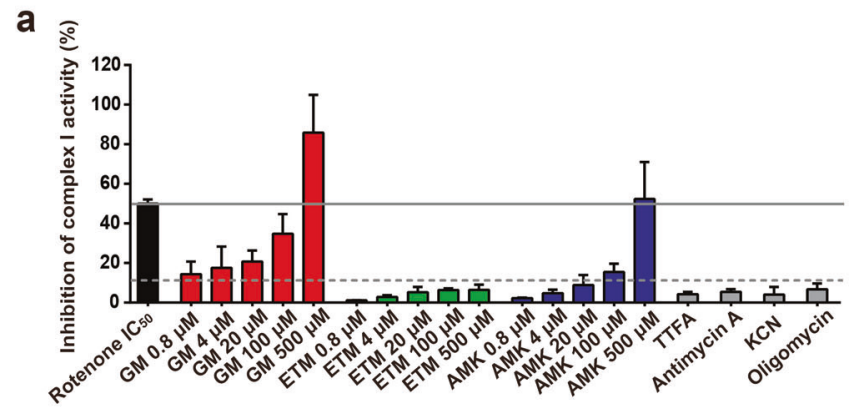

C

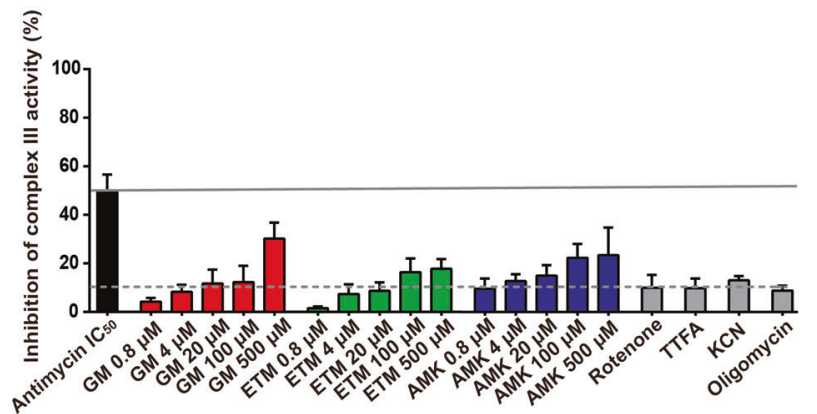

b

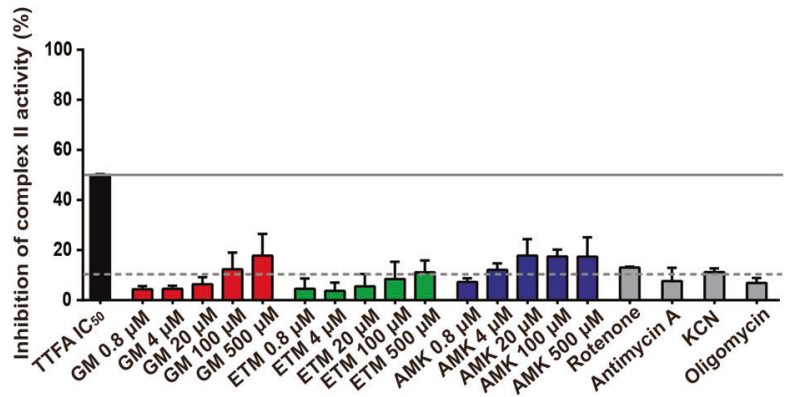

d

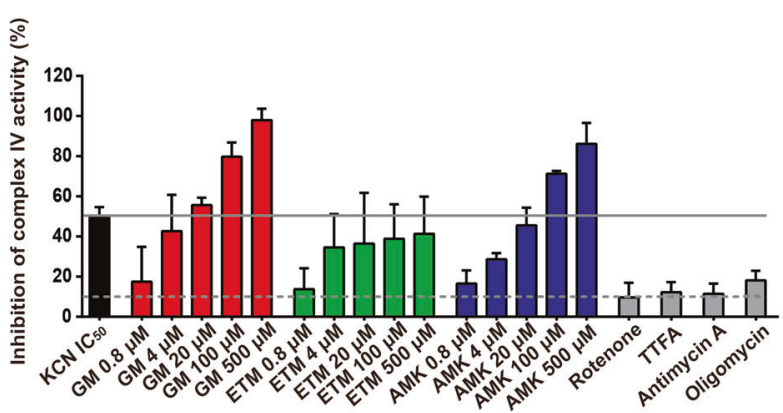

e

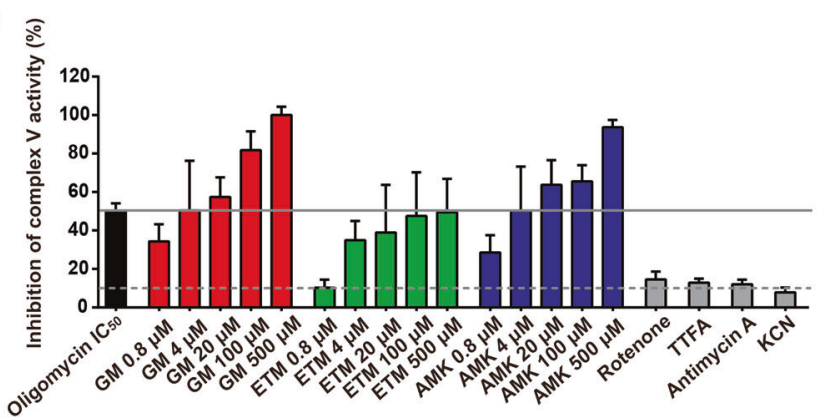

Fig. 7 Effect of AGs on the function of isolated electron transport chain (ETC) complexes. The effects of GM, ETM, and AMK (0.8, 4, 20, 100, and $500 \mu \mathrm{M}$ ) or the $\mathrm{IC}_{50} \mathrm{~S}$ of rotenone, TTFA, antimycin, KCN, and oligomycin, which are known inhibitors of complexes I, II, III, IV, and V, respectively, on the function of individual, isolated ETC protein complexes were measured. The dashed gray line represents the maximum inhibition (10\%) seen across several independent negative controls. The solid gray line represents the $\mathrm{IC}_{50}$ (median inhibitory concentration) of the positive control drugs shown to inhibit specific target complexes. The results are presented as the means \pm SEM $(n=3)$.

accumulated in the mitochondria, and ETM had a lower mitochondrial concentration than those of the GM and AMK high-concentration-treated groups. According to the endosymbiotic theory, mitochondria originate from free-living, aerobic bacteria [33]. It is likely that AGs target mitochondria and mitochondrial components by a similar mechanism of action as bacteria.

Thus, we investigated the degree of injury to mitochondria induced by GM, ETM, and AMK. The mitochondrial morphological injury changes were mild in the ETM group compared with those of the GM group or AMK high-dose group. Remarkably, the mitochondrial damage differences among the groups were more apparent after AG withdrawal. In mammalian cells, mitochondria are the major source of intracellular ROS. Therefore, we tested the hypothesis that AGs uniquely impair the mitochondrial ETC, leading to the observed overproduction of ROS and mitochondrial superoxide. We performed immunocapture-based OXPHOS activity assays to measure the perturbation in the activity of individual ETC components by the AGs and concluded that GM and AMK concentration-dependently inhibited mitochondrial ETC complexes I, IV, and V, while ETM mainly impaired the activities of complexes IV and $\mathrm{V}$ in a nonconcentration-dependent manner within the range tested. The generation of ROS has been proposed to be the principle mechanism underlying the nephrotoxicity and ototoxicity of AGs [34-36]. Recently, Jankauskas et al. [37] described that mitochondria-associated oxidative stress played a key role in the development of AG-induced nephrotoxicity and ototoxicity. In our experiment, we also concluded that GM, AMK, and ETM increased the production of ROS and mitochondrial superoxide, while ETM caused minimal damage.

To further evaluate mitochondrial function, we selected other indicators, such as MMP, ATP levels, and mtDNA copy number. It has been demonstrated that the collapse of the MMP represents mitochondrial dysfunction and results in the opening of the mitochondrial permeability transition pores, which allows apoptotic factors from the mitochondria to be released into the cytosol [38]. The disruption in MMP leads to cytochrome $c$ release from the mitochondria and increases the expression of apoptotic proteins in HK-2 cells in a diabetic nephropathy model [39]. Additionally, MMP disruption caused by AMK was reported in the mid-portion of cochlear explants [40]. Our results demonstrated that AGs indeed reduced the MMP, and ETM had the lowest effect among those tested. Additionally, mitochondria supply energy to cells and produce ATP by using oxidative phosphorylation. The kidney is only second to the heart in mitochondrial abundance, owing to its function, and so it consumes a large amount of energy supplied as ATP. 

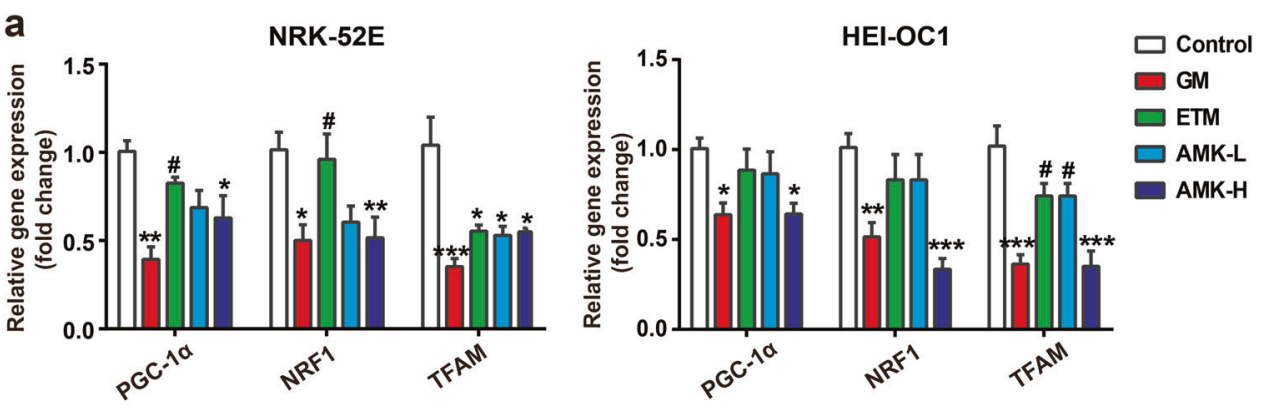

b

Control GM ETM AMK-L AMK-H

Control GM ETM AMK-L AMK-H
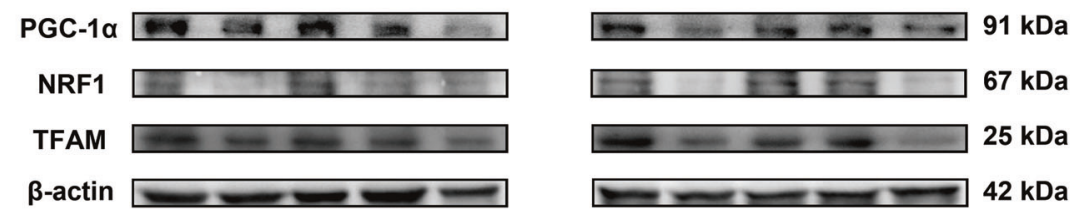

NRK-52E
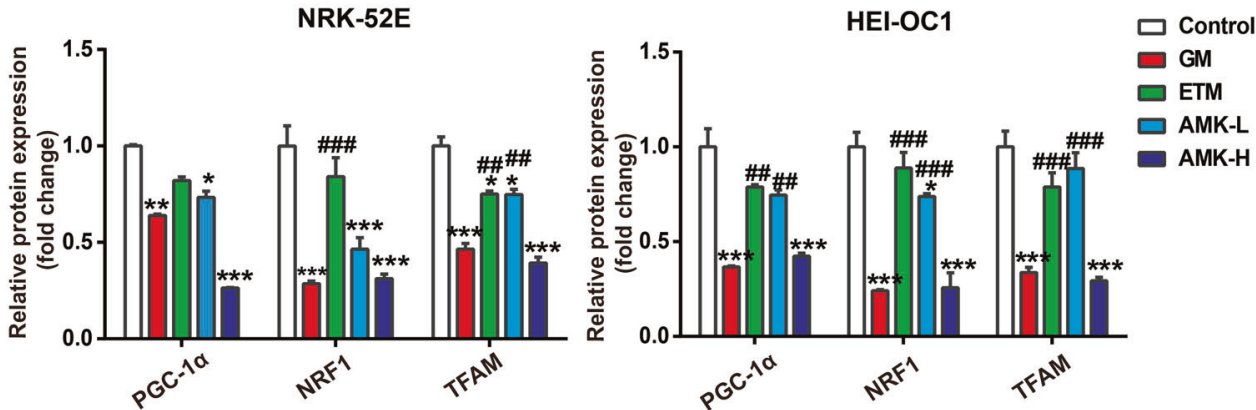

Fig. 8 AG treatment downregulated the PGC-1a-NRF1-TFAM signaling pathway and subsequently inhibited mitochondrial biogenesis. Cells in the GM, ETM, and AMK-L groups were treated with a concentration of $3 \mathrm{mM}$ for $48 \mathrm{~h}$; the AMK-H group was treated with a concentration of $9 \mathrm{mM}$ for $48 \mathrm{~h}$. a Gene expression of PGC-1 $\alpha$, NRF1, and TFAM was determined by real-time PCR; $\mathbf{b}$ protein expression of PGC$1 \alpha$, NRF1, and TFAM was determined by Western blot analysis. The results are presented as the means \pm SEM $(n=3)$. ${ }^{*} P<0.05,{ }^{* *} P<0.01,{ }^{* * *} P$ $<0.001$ vs. control group. ${ }^{\#} P<0.05,{ }^{\# \#} P<0.01,{ }^{\# \# \# P} P<0.001$ vs. GM-treated group.

Disruption in this supply can cause renal dysfunction [41]. Hair cells are cells that are specialized to sense sound vibrations, which require ATP as a neurotransmitter to maintain the physiological function [42]. Intracellular ATP depletion has been shown to induce apoptosis in primary mouse proximal tubule cells [43] and HEl-OC1 cells [44]. We confirmed the depletion of ATP after AG treatment, and ETM caused minimal damage. In addition, mitochondrial biogenesis is a complicated process that requires the synthesis, import, and incorporation of proteins and lipids, as well as the replication of mtDNA [45]. It is believed that higher mtDNA copy number is protective for cells, and some investigators have suggested that mtDNA copy number might be a surrogate marker of mitochondrial function [46]. Indeed, a case-control study found that mtDNA changes preceded bioenergetic dysfunction in patients with diabetic nephropathy [47], and mtDNA deletion has been shown to be involved in agerelated hearing loss [48]. Our findings demonstrated that AGs downregulated mtDNA copy number in NRK-52E and HEI-OC1 cells, and ETM had the least effect on this endpoint. Taken together, these results suggest that AGs directly impair mitochondrial ETC complexes and affect mitochondrial function. The low toxicity of ETM may be attributed to its slight impact on mitochondrial OXPHOS activity and function.

PGC-1a has been considered to be a central regulator of mitochondrial biogenesis [49]. PGC-1a transactivates NRF1, which in turn activates TFAM, which regulates mtDNA transcription and replication [50]. It has been well documented that PGC-1a pathways are associated with several kinds of kidney damage, including ischemia-reperfusion injury [51], diabetic nephropathy
[39], and adriamycin-induced podocyte injury [52]. However, the impacts of AGs on PGC-1a pathways are rarely reported. Consistent with the reduced mtDNA copy number and ATP production, our results also indicated that $A G$ treatment resulted in the downregulation of the PGC-1a-NRF1-TFAM pathway, suggesting that mitochondrial biogenesis is likely impaired by AGs. ETM inhibited this pathway the least.

Possible mechanisms of AG-induced nephrotoxicity and ototoxicity are depicted in Fig. 9. AGs not only directly inhibit mitochondrial complexes but also repress mitochondrial biosynthesis through the PGC-1a-NRF1-TFAM pathway.

In summary, our results showed that the low nephrotoxicity and ototoxicity of ETM are consistent with its pharmacokinetic characteristics in toxicity target organs and mitochondria, as well as the little impact on mitochondrial function. Furthermore, ETM shows the most potent activity among the AGs tested against gram-positive and gram-negative bacteria. These findings provide new insights into the use of ETM, which has demonstrated effective antibacterial activities with minimal adverse side effects and provides a new strategy for screening and evaluating AGs with high efficacy and low toxicity.

\section{ACKNOWLEDGEMENTS}

We gratefully acknowledge the kind assistance of Dr. Xia Gao and Dr. Ling Lu (Department of Otolaryngology, Nanjing Drum Tower Hospital, Clinical College of Nanjing Medical University) in the evaluation of animal ototoxicity. This work was supported by the National Natural Science Foundation of China (81573496, 81773989, 81530098, and 81573494), the China "Creation of New Drugs" Key 


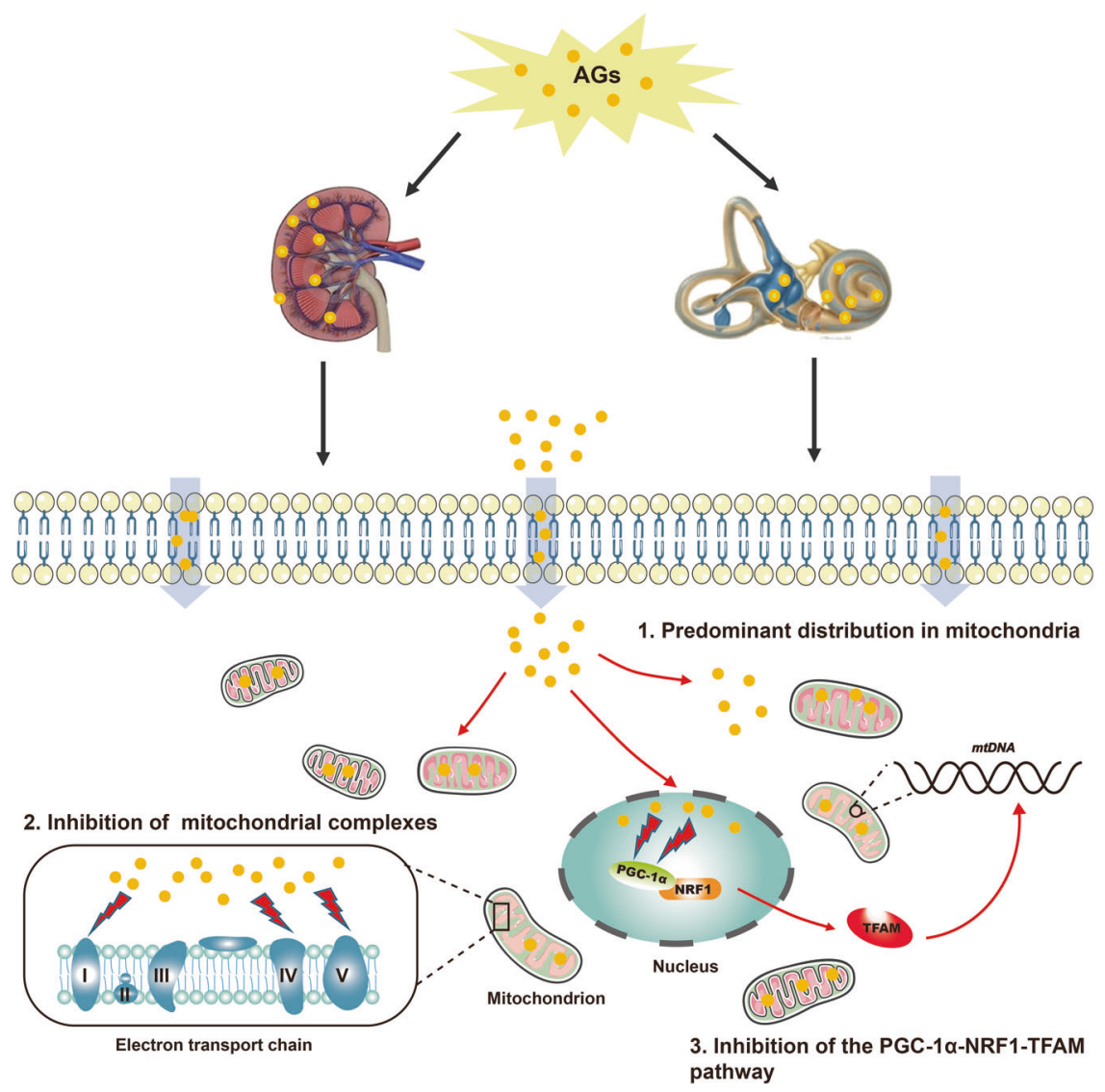

Fig. 9 Schematic representation of possible mechanisms of AG-induced nephrotoxicity and ototoxicity. The schematic mainly includes three parts: (1) AGs are predominantly distributed in the mitochondria of renal tubular cells and inner ear hair cell-like cells; (2) AGs inhibit mitochondrial ETC complexes I, IV, and V; and (3) AGs inhibit the PGC-1 $\alpha$-NRF1-TFAM pathway, which may repress mitochondrial biosynthesis.

Technology Projects (2015ZX09501001, 2017ZX09304021), the National Science and Technology Major Project of China (2019ZX09721001-006-005), the International Science and Technology Cooperation Program of China (2017YFE0109600), the Foundation for Innovative Research Groups of the National Natural Science Foundation of China (No. 81421005), the Natural Science Foundation of Jiangsu Province (No. BK20160076), the six talent peaks project in Jiangsu Province (YY-060), and the "Double First-Class" University project (CPU2018GF01).

\section{AUTHOR CONTRIBUTIONS}

LY and JWZ performed most of the experiments and analyzed the data; LY drafted the manuscript; $B C, M M C$, and QZW performed some of the experiments; DF and XYW assisted with the experiments; and FZ, GJW, YWZ, and JGS. designed the study and revised the manuscript. All of the authors have read and approved the final manuscript.

\section{ADDITIONAL INFORMATION}

The online version of this article (https://doi.org/10.1038/s41401-019-0342-5) contains supplementary material, which is available to authorized users.

Competing interests: The authors declare no competing interests.

\section{REFERENCES}

1. Becker B, Cooper MA. Aminoglycoside antibiotics in the 21 st century. ACS Chem Biol 2013;8:105-15.

2. Jackson J, Chen C, Buising K. Aminoglycosides: how should we use them in the 21st century? Curr Opin Infect Dis 2013;26:516-25.

3. Craig WA. Optimizing aminoglycoside use. Crit Care Clin 2011;27:107-21.

4. Ramirez MS, Tolmasky ME. Aminoglycoside modifying enzymes. Drug Resist Updates 2010;13:151-71.
5. Simon VK, Mosinger EU, Malerczy V. Pharmacokinetic studies of tobramycin and gentamicin. Antimicrob Agents Chemother 1973;3:445-50.

6. Bailey DN, Briggs JR. Gentamicin and tobramycin binding to human serum in vitro. J Anal Toxicol 2004;28:187-9.

7. Lode $\mathrm{H}$, Grunert $\mathrm{K}$, Koeppe $\mathrm{P}$, Langmaack $\mathrm{H}$. Pharmacokinetic and clinical studies with amikacin, a new aminoglycoside antibiotic. J Infect Dis 1976;134:S316-22.

8. Rougier F, Claude D, Maurin M, Maire P. Aminoglycoside nephrotoxicity. Curr Drug Targets Infect Disord 2004;4:153-62.

9. Guthrie OW. Aminoglycoside induced ototoxicity. Toxicology 2008;249:91-6.

10. Martinez SC, Lopez FJ, Lopez JM. Glomerular nephrotoxicity of aminoglycosides. Toxicol Appl Pharmacol 2007;223:86-98.

11. Zhao C, Li J, Hou J, Guo M, Zhang Y, Chen Y. A randomized controlled clinical trial on etimicin, a new aminoglycoside antibiotic, versus netilmicin in the treatment of bacterial infections. Chin Med J 2000;113:1026-30.

12. Chaudhary M, Kesava NG, Kumar S, Payasi A. Comparative antibacterial activity of a novel semisynthetic antibiotic: etimicin sulphate and other aminoglycosides. World J Microbiol Biotechnol 2012;28:3365-71.

13. Lan TC, Lin HJ, Ping YH, Ming LJ. Comparison of clinical efficacy and safety between etimicin and netilmicin in treatment of bacterial infections in lower respiratory tract. Chin J New Drugs Clin Rem 2004;23:83-7.

14. Payasi A, Chaudhary M, Gupta A, Dwivedi VK. Sub-acute toxicity study of a new aminoglycoside etimicin sulphate in swiss albino mice. J Toxicol Sci 2010;35:479-84.

15. Yao L, Zhou F, Cai M, Peng Y, Sun J, Chen Q, et al. Development and validation of a sensitive LC-MS/MS method without derivatization/ion-pairing agents for etimicin quantification in rat plasma, internal ear and kidney. J Pharm Biomed Anal 2017;146:96-102.

16. Kalinec GM, Webster $P$, Lim DJ, Kalinec F. A cochlear cell line as an in vitro system for drug ototoxicity screening. Audio Neurootol 2003;8:177-89.

17. Zhang J, Zhou F, Wu X, Zhang X, Chen Y, Zha BS, et al. Cellular pharmacokinetic mechanisms of adriamycin resistance and its modulation by $20(S)$-ginsenoside Rh2 in MCF-7/Adr cells. Br J Pharmacol 2012;165:120-34. 
18. Yue R, Xia X, Jiang J, Yang D, Han Y, Chen X, et al. Mitochondrial DNA oxidative damage contributes to cardiomyocyte ischemia/reperfusion-injury in rats: cardioprotective role of lycopene. J Cell Physiol 2015;230:2128-41.

19. Timmeren MM, Heuvel MC, Bailly V, Bakker SJ, Goor H, Stegeman CA. Tubular kidney injury molecule-1 (KIM-1) in human renal disease. J Pathol 2007;212:209-17.

20. Shang W, Wang Z. The update of NGAL in acute kidney injury. Curr Protein Pept Sci 2017;18:1211-7.

21. Chen CS, Aberdeen GC. The sensitive period for induction of susceptibility to audiogenic seizures by kanamycin in mice. Arch Otorhinolaryngol 1981;232:215-20.

22. Nadanaciva S, Bernal A, Aggeler R, Capaldi R, Will Y. Target identification of drug induced mitochondrial toxicity using immunocapture based OXPHOS activity assays. Toxicol In vitro 2007;21:902-11.

23. Nadanaciva S, Dykens JA, Bernal A, Capaldi RA, Will Y. Mitochondrial impairment by PPAR agonists and statins identified via immunocaptured OXPHOS complex activities and respiration. Toxicol Appl Pharmacol 2007;223:277-87.

24. Scarpulla RC. Transcriptional paradigms in mammalian mitochondrial biogenesis and function. Physiol Rev 2008;88:611-38.

25. Christensen EF, Reiffenstein JC, Madissoo H. Comparative ototoxicity of amikacin and gentamicin in cats. Antimicrob Agents Chemother 1977;12:178-84.

26. Olier B, Morin JP, Thomas N, Fillastre JP. Habekacin: a new aminoglycoside. Study of nephrotoxicity in rats in comparison with gentamicin, netilmicin and amikacin. Pathol Biol 1988;36:795-800.

27. Yamamoto $M$, Kurata $Y$, Chiba S. Effects of aminoglycoside antibiotics on the auditory brainstem response and post rotatory nystagmus in rats. Fundam Appl Toxicol 1992;18:499-503.

28. Lopez JM, Quiros Y, Vicente L, Morales Al, Lopez FJ. New insights into the mechanism of aminoglycoside nephrotoxicity: an integrative point of view. Kidney Int 2011;79:33-45.

29. Jiang M, Karasawa T, Steyger PS. Aminoglycoside-induced cochleotoxicity: a review. Front Cell Neurosci 2017;11:308. https://doi.org/10.3389/ fncel.2017.00308.

30. Toyoda Y, Tachibana M. Tissue levels of kanamycin in correlation with oto- and nephrotoxicity. Acta Otolaryngol 1978;86:9-14.

31. Steyger PS, Peters SL, Rehling J, Hordichok A, Dai CF. Uptake of gentamicin by bullfrog saccular hair cells in vitro. J Assoc Res Otolaryngol 2003:4:565-78.

32. Beauchamp D, Gourde $P$, Bergeron MG. Subcellular distribution of gentamicin in proximal tubular cells, determined by immunogold labeling. Antimicrob Agents Chemother 1991;35:2173-9.

33. Gray MW, Burger G, Lang BF. Mitochondrial evolution. Science 1999;283:1476-81.

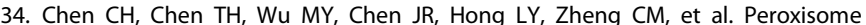
proliferator-activated receptor alpha protects renal tubular cells from gentamicininduced apoptosis via upregulating $\mathrm{Na}^{+} / \mathrm{H}^{+}$exchanger NHE1. Mol Med 2016;21:886-9.

35. Balaban RS, Nemoto S, Finkel T. Mitochondria, oxidants, and aging. Cell 2005;120:483-95.
36. Huang T, Cheng AG, Stupak H, Liu W, Kim A, Staecker H, et al. Oxidative stressinduced apoptosis of cochlear sensory cells: otoprotective strategies. Int J Dev Neurosci 2000;18:259-70.

37. Jankauskas SS, Plotnikov EY, Morosanova MA, Pevzner IB, Zorova LD, Skulachev VP, et al. Mitochondria-targeted antioxidant SkQR1 ameliorates gentamycininduced renal failure and hearing loss. Biochemistry 2012;77:666-70.

38. Mao WP, Ye JL, Guan ZB, Zhao JM, Zhang C, Zhang NN, et al. Cadmium induces apoptosis in human embryonic kidney (HEK) 293 cells by caspase-dependent and caspase-independent pathways acting on mitochondria. Toxicol In vitro 2007;21:343-54.

39. Xiao L, Zhu X, Yang S, Liu F, Zhou Z, Zhan M, et al. Rap1 ameliorates renal tubular injury in diabetic nephropathy. Diabetes 2014;63:1366-80.

40. Kim YR, Kim MA, Cho HJ, Oh SK, Lee IK, Kim UK, et al. Galangin prevents aminoglycoside-induced ototoxicity by decreasing mitochondrial production of reactive oxygen species in mouse cochlear cultures. Toxicol Lett 2016;245:78-85.

41. Hall AM, Unwin RJ. The not so 'mighty chondrion': emergence of renal diseases due to mitochondrial dysfunction. Nephron Physiol 2007;105:1-10.

42. Wang HC, Lin CC, Cheung R, Zhang-Hooks $Y$, Agarwal A, Ellis-Davies G, et al. Spontaneous activity of cochlear hair cells triggered by fluid secretion mechanism in adjacent support cells. Cell 2015;163:1348-59.

43. Lieberthal W, Menza SA, Levine JS. Graded ATP depletion can cause necrosis or apoptosis of cultured mouse proximal tubular cells. Am J Physiol 1998;274: F315-27.

44. Hayashi K, Dan K, Goto F, Tshuchihashi N, Nomura Y, Fujioka M, et al. The autophagy pathway maintained signaling crosstalk with the Keap1-Nrf2 system through p62 in auditory cells under oxidative stress. Cell Signal 2015;27:382-93.

45. Hock MB, Kralli A. Transcriptional control of mitochondrial biogenesis and function. Annu Rev Physiol 2009;71:177-203.

46. Cho YM, Park KS, Lee HK. Genetic factors related to mitochondrial function and risk of diabetes mellitus. Diabetes Res Clin Pract 2007;77:S172-7.

47. Czajka A, Ajaz S, Gnudi L, Parsade CK, Jones P, Reid F, et al. Altered mitochondrial function, mitochondrial DNA and reduced metabolic flexibility in patients with diabetic nephropathy. EBioMedicine 2015;2:499-512.

48. Chen $\mathrm{H}$, Tang J. The role of mitochondria in age-related hearing loss. Biogerontology 2014;15:13-9.

49. Wu Z, Puigserver $P$, Andersson $U$, Zhang C, Adelmant G, Mootha V, et al. Mechanisms controlling mitochondrial biogenesis and respiration through the thermogenic coactivator PGC-1. Cell 1999;98:115-24.

50. Scarpulla RC. Nuclear activators and coactivators in mammalian mitochondrial biogenesis. Biochim Biophys Acta 2002;1576:1-14.

51. Garrett SM, Whitaker RM, Beeson CC, Schnellmann RG. Agonism of the 5hydroxytryptamine $1 \mathrm{~F}$ receptor promotes mitochondrial biogenesis and recovery from acute kidney injury. J Pharmacol Exp Ther. 2014;350:257-64.

52. Zhu C, Xuan X, Che R, Ding G, Zhao M, Bai M, et al. Dysfunction of the PGC1alpha-mitochondria axis confers adriamycin-induced podocyte injury. Am J Physiol Ren Physiol 2014;306:F1410-7. 